\title{
Comparison of the Surface Coat Proteins of the Pine Wood Nematode Appeared During Host Pine Infection and In Vitro Culture by a Proteomic Approach
}

\author{
Ryoji Shinya, Hironobu Morisaka, Yuko Takeuchi, Mitsuyoshi Ueda, and Kazuyoshi Futai
}

First author: Research Fellow of the Japan Society for the Promotion of Science; first, third, and fifth authors: Division of Environmental Science and Technology, Graduate School of Agriculture, Kyoto University, Kyoto, Japan; and second and fourth authors: Division of Applied Life Sciences, Graduate School of Agriculture, Kyoto University, Kyoto, Japan.

Accepted for publication 29 June 2010.

\begin{abstract}
Shinya, R., Morisaka, H., Takeuchi, Y., Ueda, M., and Futai, K. 2010. Comparison of the surface coat proteins of the pine wood nematode appeared during host pine infection and in vitro culture by a proteomic approach. Phytopathology 100:1289-1297.

Pine wilt disease, caused by the pine wood nematode (PWN), Bursaphelenchus xylophilus, has become of worldwide quarantine concern in recent years. Here, we disclosed the surface coat (SC) proteins of the PWN which are thought to be one of the key components in pine wilt development. This is the first report that focused on the SC proteins and thoroughly identified those proteins of a plant-parasitic nematode using

proteins drastically increased during infection of the host pine. Thirtyseven protein bands showed significant quantity differences between fungus-grown and host-origin PWNs, and were used for identification by matrix-assisted laser desorption ionization time of flight mass spectrometry analysis. These included several proteins that are presumed to be involved in the host immune response; for example, regulators of reactive oxygen species (ROS) and a ROS scavenger. These results might suggest that the PWN SC proteins are crucial in modulating or evading host immune response. Our data provide a new insight into the mechanism of pine wilt disease and the biological role of the SC proteins of plantparasitic nematodes.
\end{abstract} the proteomic approach. In this study, SC protein profiles were compared for PWNs grown on the fungus Botrytis cinerea and in host pine seedlings. The results demonstrated that the gross amount of PWN SC
Additional keywords: Pinus thunbergii, proteome.
Pine wilt disease (PWD), caused by the pine wood nematode (PWN), Bursaphelenchus xylophilus, is one of the most serious tree diseases in the world, not only because pine forests are important sources of forest products but also because pine trees are used in wind, tide, and sand breaks; landslide prevention; and beautiful landscapes, especially in East Asia. However, pine forests now face a catastrophe resulting from PWD. The disease was first discovered in the early 20th century in Japan and, in 1971 , B. xylophilus was shown to cause the disease (20). It is now known that the PWN originated from the United States and introduced into Japan from there. Subsequently, in the 1980s, the pathogen spread to neighboring East Asian countries and, since then, has been found causing pine wilt in Portugal in 1999 (27) and in Spain in 2008. Thus, the disease has become a worldwide threat. As a result of international trade in forest products, the PWN poses a potentially serious problem in both the Southern and Northern Hemispheres. The European and Mediterranean Plant Protection Organization has listed both the PWN and its vector, the Monochamus beetle, as quarantine pest A1.

Resulting from research on PWD over the years, several hypotheses have been put forward regarding the pathogenic mechanisms for the disease (e.g., several pathogen-produced molecules have been suggested as playing key roles in PWN-host interactions). These include, for example, cell-wall-degrading enzymes secreted by PWN $(17,18,28)$ and surface coat (SC) proteins of PWN (39). Also, it has been reported that molecules

Corresponding author: R. Shinya; E-mail address: r.shinya@fx5.ecs.kyoto-u.ac.jp

doi:10.1094/PHYTO-04-10-0109

(C) 2010 The American Phytopathological Society originating from $\mathrm{PWN}$-associated bacteria are important in PWD development $(12,40)$. Furthermore, it has been shown that the abnormal regulation of reactive oxygen species (ROS) which are produced in large quantities in pine trees during the initial stage of PWD infection are a key factor in the pine wilt mechanism (13). However, just how much each of these factors contributes in the development of PWD still remains unclear. This situation is caused not only by the ecological complexity of PWD but also by the lack of genomic information and tools for doing functional genomic analysis on the organisms involved in PWD.

Here, we analyzed the PWN SC proteins based on the $B$. xylophilus expressed sequence tag (EST) information recently constructed (16) to clarify the detailed profile of the SC proteins by a proteomic approach. Many reports suggest that nematode SC plays various crucial roles in host-parasite interactions $(31,36)$. For example, some of the many functions of SC are regulation of other microbes' adhesion to the nematode's body surface, lubrication, elicitation of the host defense responses, and modulation to help counter host defense responses $(3,8,10,11,36)$. However, to date, little has been discovered about the detailed characteristics of the nematode SC because of difficulties involved in dealing with, for example, insufficient quantity, and heavy glycosylation. In particular, there have been no reports of attempts to thoroughly identify the SC proteins of plant-parasitic nematodes. The reasons for this vary but two major problems occur because of the nematodes are very small and difficult to propagate on a large scale.

In the present study, we identified the series of SC proteins which are specifically regulated in relation to the PWN infection into host pine trees by comparing the SC proteins between PWNs grown on a fungus versus those propagated in pine seedlings. 
Identification of these proteins should provide a new insight into the biological role of SC proteins of plant-parasitic nematodes, and allow us to propose the roles of those molecules in host pinePWN interactions.

\section{MATERIALS AND METHODS}

Nematodes and their propagation. The virulent ' $\mathrm{S} 10$ ' isolate (originally isolated from Shimane Prefecture, Japan) of $B$. xylophilus was used in all the experiments. To compare the profiles of SC proteins, the nematodes were propagated on either the fungus Botrytis cinerea, designated "nematodes grown on the fungus" (NGFs), or the host, Japanese black pine (Pinus thunbergii) seedlings, designated "nematodes grown on the pine seedlings" (NGPs). The NGFs were cultured on B. cinerea growing on autoclaved barley grains and incubated at $25^{\circ} \mathrm{C}$ in $50-\mathrm{ml}$ Erlenmeyer flasks. After incubation for 18 days, the PWNs were extracted for $12 \mathrm{~h}$ from the culture using the Baermann funnel technique (6). PWNs for use in inoculating the pine seedlings were also cultured on $B$. cinerea for 14 days before being inoculated into 3-year-old, potted, Japanese black pine, $P$. thunbergii, seedlings kept outdoors at Kyoto University, Kyoto, Japan, during the summers of 2008 and 2009. After 18 to 22 days from PWN inoculation, the PWN population was extracted from woody pieces of the inoculated seedlings using the Baermann funnel technique for $12 \mathrm{~h}$, to serve as the NGP sample. Both samples of nematodes (NGFs and NGPs) were used for the extraction of SC proteins.

Extraction of SC proteins. The SC proteins of the PWN were extracted using the standard procedure for extraction of the SC of nematodes $(24,32)$, modified as follows. To compare the gross quantities of the proteins extracted from NGF and NGP, $8 \times$ $10^{5}$ nematodes (mixed life stages) were suspended in $500 \mu \mathrm{l}$ of 2\% 3-[(3-cholamidopropyl)-dimethylammonio]-1-propanesulfonate (CHAPS) in phosphate-buffered saline (PBS) with $1 \%$ protease inhibitor cocktail for mammalian cells (Sigma-Aldrich, St. Louis), and gently agitated for $1 \mathrm{~h}$ at $4{ }^{\circ} \mathrm{C}$. The adequacy of this protocol was confirmed by electron microscope, and the PWN lived and had high activity even after the extraction of SC protein. The nematodes were then pelletized by centrifugation at $3,000 \times g$ for $5 \mathrm{~min}$ at $25^{\circ} \mathrm{C}$ and the supernatant was collected. Next, for comparative proteomic analysis of SC proteins, $2 \times 10^{7}$ nematodes (mixed life stages) propagated under the two different conditions were suspended in $5 \mathrm{ml}$ of $2 \%$ CHAPS in PBS with $1 \%$ protease inhibitor cocktail, and the supernatant was collected in the same manner as described above.

Protein sample preparation and quantification. Each protein solution that was obtained was desalted and buffer exchanged on PD-10 columns (GE Healthcare, Piscataway, NJ) with $20 \mathrm{mM}$ Tris-HCl ( $\mathrm{pH} 8.0)$ according to the manufacturer's protocol. Then, each protein concentration was determined using a Protein Assay Bichinchoninate (BCA) kit (Nacalai Tesque, Kyoto, Japan) according to the manufacturer's protocol. The protein concentration data for three replicates were subjected to evaluation for statistical significance with analysis of variance, followed by Tukey's highly significant difference test.

Protein separation by reversed-phase high-performance liquid chromatography and sodium dodecyl sulfate polyacrylamide gel electrophoresis. The extracted and desalted mass proteins of NGFs and NGPs were separated by reversed-phase high-performance liquid chromatography (RP-HPLC) as a firstdimension protein separation. Before being submitted to RPHPLC analysis, each protein amount was equally adjusted to $90 \mu \mathrm{g}$ for the differential analysis. An HPLC system consisted of an LC-20AD pump (Shimadzu, Kyoto, Japan), 7725 injector (Rheodyne, Cotati, CA), CTO-20A column oven (Shimadzu), and SPD-M20A UV detector (Shimadzu). The chromatographic data were collected and processed by LC solution software (Shi- madzu). In RP-HPLC analysis for protein separation, a COSMOSIL Protein-R (15 cm long, $4.6 \mathrm{~mm}$ i.d.) (Nacalai Tesque) was used at a flow rate of $1.0 \mathrm{ml} / \mathrm{min}$. The gradient was provided by changing the mixing ratio of the two eluents: $\mathrm{A}, 0.1 \%$ ( $\mathrm{vol} / \mathrm{vol}$ ) trifluoroacetic acid (TFA), and $\mathrm{B}$, acetonitrile (ACN) containing $0.1 \%$ ( vol $/ \mathrm{vol}$ ) TFA. The gradient was started with $10 \% \mathrm{~B}$ for $10 \mathrm{~min}$, increased to $60 \% \mathrm{~B}$ for $10 \mathrm{~min}$, and further increased to $100 \% \mathrm{~B}$ within 2 min to wash the column. The fractions including separated proteins were collected from 8 to $11.8 \mathrm{~min}$ at 20 -s intervals and from 11.8 to $14.75 \mathrm{~min}$ at 9 -s intervals. Thirty-three fractions (1 to $10, \mathrm{~A}$ to $\mathrm{W}$ ) were collected during RP-HPLC analysis. These fractions were dried by vacuum centrifugation and resuspended in $10 \mu \mathrm{l}$ of PBS ( $\mathrm{pH}$ 7.4). The resuspended samples were mixed with an equal volume of $2 \times$ sodium dodecyl sulfate (SDS) sample buffer and heated at $100^{\circ} \mathrm{C}$ for 3 min before being loaded onto a gel for subsequent SDS-polyacrylamide gel electrophoresis (PAGE) analysis.

Tricine SDS-PAGE and Laemmli SDS-PAGE systems were used as the second-dimension protein separations. Fractions 1 to 10 were separated by a Tricine SDS-PAGE system using $15 \%$ Tris-tricine gels (e-PAGEL; ATTO, Tokyo) in a standard minislab PAGE apparatus (model AE-6500; ATTO) to separate low molecular weight proteins. Low-Range Rainbow Molecular Weight Markers (GE Healthcare) were used to determine the molecular mass of the proteins. On the other hand, fractions A to $\mathrm{W}$ were separated by Laemmli SDS-PAGE system using 5 to $20 \%$ gradient gels (e-PAGEL; ATTO). Full-Range Rainbow Molecular Weight Markers (GE Healthcare) were used to determine the molecular mass of the proteins. The proteins were silver stained using a Sil-Best-staining kit (Nacalai Tesque) according to the manufacturer's instructions.

Gel band visualization and relative quantification. ImageJ software (National Institutes of Health, version 1.42) was used for relative quantitative densitometric analysis of gel band intensities. Selected bands were quantified based on their relative intensities. Relative fold-change was calculated for gel band pairs based on the relative intensities between proteins of NGF and NGP. The densitometry value data for three independent replicates of NGF and NGP samples were subjected a Student's $t$ test. A $P$ value < 0.05 was considered a significant difference for the NGF and NGP samples.

Tryptic in-gel digestion. In-gel digestion was done as follows. Briefly, protein bands with significant differences between samples were excised and destained by a Sil-Best Destain Kit (Nacalai Tesque) according to the manufacturer's protocol. The destained gels were dehydrated in $100 \mu \mathrm{l}$ of ACN and completely dried by vacuum centrifugation. The excised gel pieces were incubated in freshly prepared $10 \mathrm{mM}$ dithiothreitol (DTT) per 25 $\mathrm{mM}$ ammonium hydrogen carbonate $\left(\mathrm{NH}_{4} \mathrm{HCO}_{3}\right)$ at $56^{\circ} \mathrm{C}$ for $1 \mathrm{~h}$, and then the proteins were alkylated in $55 \mathrm{mM}$ iodoacetamide per $25 \mathrm{mM} \mathrm{NH}_{4} \mathrm{HCO}_{3}$ in the dark for $45 \mathrm{~min}$ at room temperature. The gel pieces were subsequently dehydrated by $200 \mu \mathrm{l}$ of $25 \mathrm{mM}$ $\mathrm{NH}_{4} \mathrm{HCO}_{3}$ in $50 \% \mathrm{ACN}$ (two times) and dried by vacuum centrifugation for $15 \mathrm{~min}$. The samples were incubated in $20 \mu \mathrm{l}$ of $50 \mathrm{mM} \mathrm{NH} \mathrm{HCO}_{3}$ containing trypsin (Promega Diagnostics, Madison, WI) at $10 \mu \mathrm{g} / \mathrm{ml}$ for $12 \mathrm{~h}$ at $37^{\circ} \mathrm{C}$. Following enzymatic digestion, the resultant peptides were extracted two times with 50 and then $25 \mu \mathrm{l}$ of $0.1 \%$ TFA in $50 \% \mathrm{ACN}$ and concentrated to 10 to $15 \mu \mathrm{l}$ by vacuum centrifugation. Furthermore, the peptides were desalted using a MonoTip C18 (GL Sciences, Tokyo) and eluted with $0.1 \%$ TFA in $50 \%$ ACN.

Matrix-assisted laser desorption ionization time-of-flight mass spectrometry analysis. A peptide cocktail was mixed with the matrix solution, consisting of $\alpha$-cyano-4-hydroxycinnamic acid at $10 \mathrm{mg} / \mathrm{ml}$ in $50 \%$ ACN containing $0.1 \%$ TFA. The mixture ( $1 \mu \mathrm{l})$ was spotted onto a matrix-assisted laser desorption ionization time-of-flight (MALDI-TOF) plate, followed by drying at room temperature. Mass spectra were obtained on a Voyager RP 
mass spectrometer (Applied Biosystems, Foster City). The mass spectrometer was calibrated using the ProteoMass peptide MALDI Calibration Kit (Sigma-Aldrich) with angiotensin II and ACTH peptide fragment.

Database searching. Mass spectrometry (MS) data were interpreted by searching the National Center for Biotechnology Information nonredundant database (NCBInr) using the MASCOT search engine on the Matrix Science public domain server (http://www.matrixscience.com). Taxonomy was first limited to "Caenorhabditis elegans" and, subsequently, set to "All entries". The search parameters were defined as follows: trypsin digest (allowing up to two missed cleavages), cysteine carbamidomethylation, and maximal mass tolerance of $0.2 \mathrm{Da}$.

Strategies of protein identification. Currently, no protein information is given for Bursaphelenchus xylophilus in any database. Also, the present EST database for B. xylophilus is not efficient to directly identify proteins in peptide mass fingerprinting (PMF). A specific strategy needed to be developed for identifying the proteins of B.xylophilus due to these limitations inherent in the incompletely sequenced or annotated information of B. xylophilus. Therefore, we applied the homology-based identification strategy of Mezhoud et al. (25). When the MS data were obtained, the NCBI protein database was searched for the candidate protein which is homologous to that of B. xylophilus using the MASCOT search engine. Then, the amino acid sequence of the candidate protein was submitted for tBLASTn search in the B. xylophilus EST database using ForestGEN server (http://forestgen.ffpri.affrc.go.jp). A protein with a significant MOWSE score $(P<0.05)$ in the MASCOT search was regarded as a hit for the candidate protein. However, in some cases, when no significant MOWSE score was observed, the protein was regarded as a hit for the candidate protein only when it fulfilled all of the following conditions: (i) at least four peptide matches were observed, (ii) the experimental molecular weight was consistent with the theoretical one, and (iii) the result of tBLASTn search in the B. xylophilus EST database showed a significant (bit score $>100$ and $e$ value $<0.001)$ hit. Then, the amino acid sequence of a candidate protein was submitted for the tBLASTn search, and we determined that the protein was successfully identified only when it showed a cluster of B. xylophilus with a significant (bit score $>100$ and $e$ value $<0.001$ ) hit. The output of the search was given as a list of clusters from B. xylophilus (Tables 1 and 2). The sequence thus obtained was used for BLASTx search in the ForestGEN server to determine its putative functions and the protein family to which it belongs.

Immunohistochemical staining. PWNs propagated on the fungus and in the pine seedlings were collected using the protocol described above and fixed with $4 \%$ paraformaldehyde in $0.1 \mathrm{M}$ phosphate buffer for $30 \mathrm{~min}$ at room temperature. The nematodes were pretreated with antigen retrieval solution HistoVT One (Nacalai Tesque) for $20 \mathrm{~min}$ at $70^{\circ} \mathrm{C}$, and then blocked in a solution of $3 \%$ bovine serum albumin in PBS for $30 \mathrm{~min}$ at room

TABLE 1. Identification of Bursaphelenchus xylophilus surface coat proteins upregulated during infection into host pine by matrix-assisted laser desorption ionization time-of-flight mass spectrometry

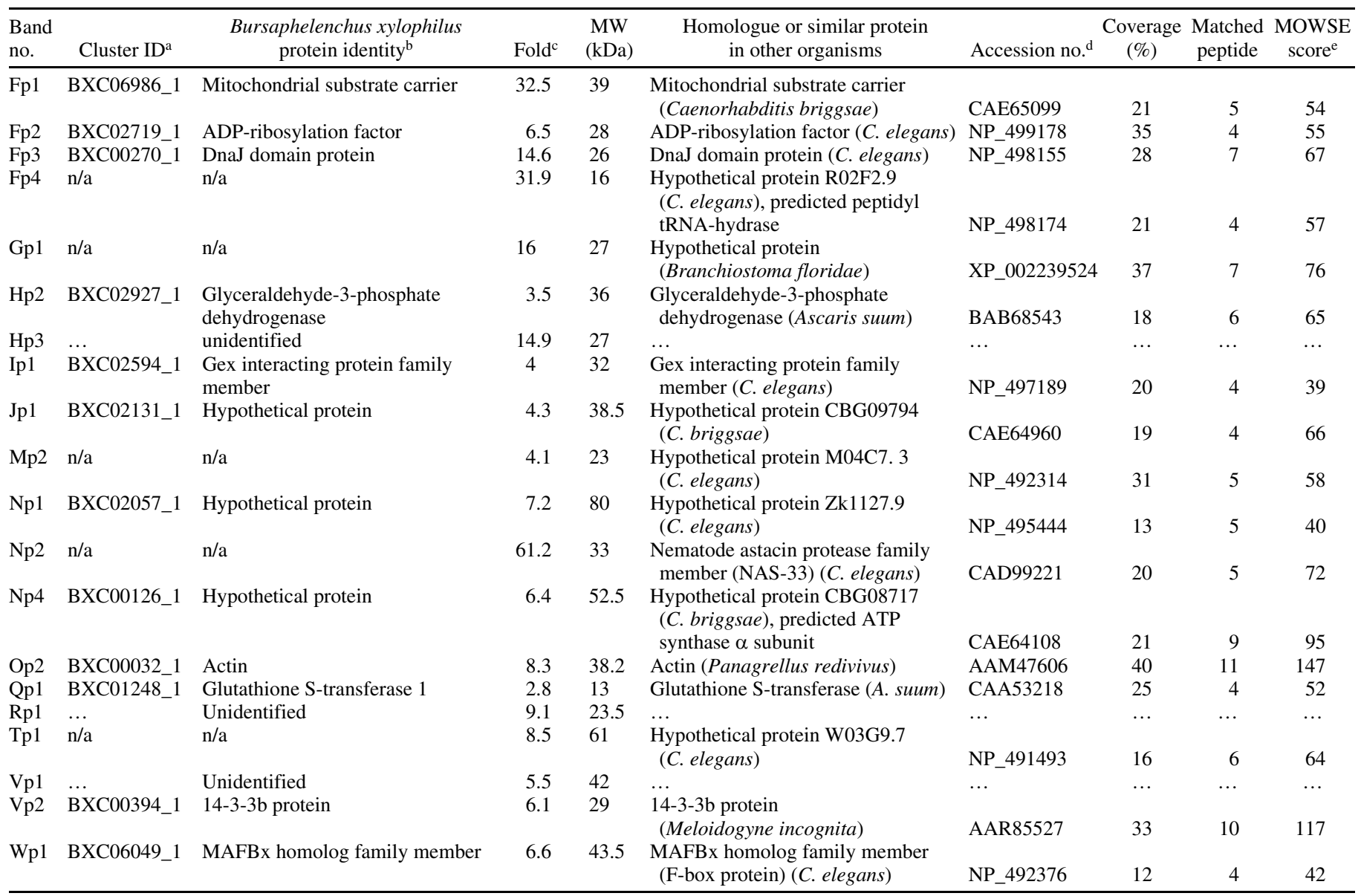

a Cluster ID given in the B. xylophilus expressed sequence tag database.

b Protein identity by homology with other nematode's entry; n/a = not available.

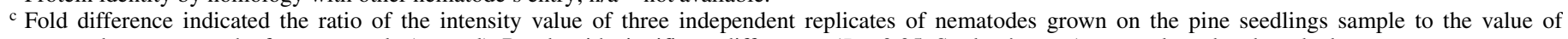
nematodes grown on the fungus sample (control). Bands with significant differences $(P<0.05$; Student's $t$ test) were selected and marked.

${ }^{\mathrm{d}}$ GenBank protein accession number.

e A MOWSE score given by MASCOT search. 
temperature. After blocking, the nematodes were incubated with rabbit polyclonal antibodies against actin (A2668; SigmaAldrich), glyceraldehydes-3-phosphate dehydrogenase (GAPDH) (G9545; Sigma-Aldrich), or 14-3-3 protein (ab6081; Abcam, Cambridge, UK) diluted at $1 / 30,1 / 100$, and $1 / 40$, respectively, in PBS overnight at $4^{\circ} \mathrm{C}$. After the incubation, the nematodes were washed three times with PBS and incubated with fluorescein isothiocyanate (FITC)-conjugated anti-rabbit immunoglobulin G (F9887; Sigma-Aldrich) diluted $1 / 160$ in PBS for $1 \mathrm{~h}$ at room temperature. They were then washed three times with PBS and observed using a confocal laser-scanning microscope (Zeiss LSM510; Carl Zeiss, Oberkochen, Germany). FITC was excited using a 488-nm argon laser line. Serial-section images were acquired using the LSM510 operation system and software. Single section images were selected from these and exported in TIFF format. To assess nonspecific staining, a negative control experiment was performed by incubating the nematodes without primary antibody or with rabbit preimmune serum diluted at $1 / 100$ in PBS for $1 \mathrm{~h}$ at $4^{\circ} \mathrm{C}$.

Scanning electron microscope observations. PWNs were propagated on both the fungus and in host pine seedlings and extracted (as previously) and prefixed in $2 \%$ formaldehyde plus $2 \%$ glutaraldehyde in $0.1 \mathrm{M}$ phosphate buffer $(\mathrm{pH} 7.2)$ for $12 \mathrm{~h}$ at $4{ }^{\circ} \mathrm{C}$. The nematodes were post-fixed with $2 \%$ osmium tetroxide for $30 \mathrm{~min}$, and then dehydrated with an ethanol series $(30,50$, 70,90 , and $95 \%$, and twice in $100 \%$ ethanol) for $30 \mathrm{~min}$ at each concentration. Then, the nematodes were dried by a critical point dryer (JCPD-5; JEOL, Tokyo) using liquid carbon dioxide, and sputter-coated with Au-Pd using a magnetron sputter (MSP-1S; Vacuum Device, Ibaraki, Japan). Scanning electron microscope (SEM) images were taken using a VE-8800 (Keyence, Osaka, Japan).

\section{RESULTS}

Quantification of the total SC proteins extracted. The concentration of the gross SC proteins extracted from three replicates of $8 \times 10^{5}$ PWNs (NGF and NGP) was determined by BCA protein assay to be $9.2 \pm 3.4$ (mean \pm standard deviation) and $81.7 \pm 3.9 \mu \mathrm{g} / \mathrm{ml}$, respectively (Fig. 1). No significant difference was noted between the body surface ultrastructure of NGFs and NGPs in SEM images (data not shown). The amount of SC protein covering PWNs extracted from pine seedlings was approximately nine times larger than that of PWNs which reproduced on the fungus.

RP-HPLC and SDS-PAGE analysis. The SC protein samples were separated by a special two-dimensional protein separation system which combined RP-HPLC with SDS-PAGE. First, the proteins of NGF and NGP extracted were separated by RP-HPLC as a first-dimension protein separation The chromatograms overlaid showed remarkable differences between the NGF and NGP samples from fractions $\mathrm{F}$ to $\mathrm{H}$ (retention time: 12.03 to $12.30 \mathrm{~min}$ ) (Fig. 2). Subsequently, 33 fractions of both samples collected from each RP-HPLC experiment were paired and loaded onto an SDS-PAGE gel for the second-dimension protein separation (Fig. 3). The quantitative densitometric analysis, based on the protein band intensity, showed a significant increase for 29 gel bands in NGPs (from pine seedlings) compared with NGFs $(P<0.05$; Student's $t$ test). However, eight gel bands appeared significantly less in NGP than NGF $(P<0.05$; Student's $t$ test). As well, $\approx 100$ independent proteins without quantity difference between samples ( $P>0.05$; Student's $t$ test) were clearly observed in the images of SDS-PAGE (Fig. 3).

Overview of SC protein identification. An overview of the identification of the PWN SC proteins is shown in Figure 4. Thirty-seven gel bands which showed a significant difference in quantity between NGF and NGP (Fig. 3) were selected and excised for protein identification by MALDI-TOF/MS analysis. Of these, 29 proteins were upregulated and the other 8 were downregulated in NGP compared with NGF samples. From

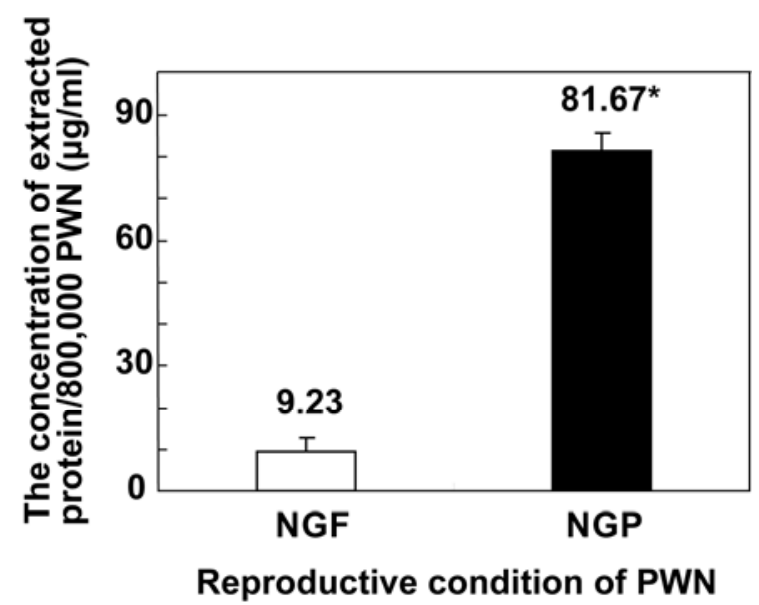

Fig. 1. Comparison of the total protein concentration extracted from Bursaphelenchus xylophilus grown on the fungus Botrytis cinerea (NGF) and on Japanese black pine seedlings (NGP). Concentrations shown are the values per $8 \times 10^{5}$ nematodes averaged from three replicates. The asterisk denotes a significant difference between NGF and NGP $(P<0.01$, Tukey's test $)$. PWN $=$ pine wood nematode.

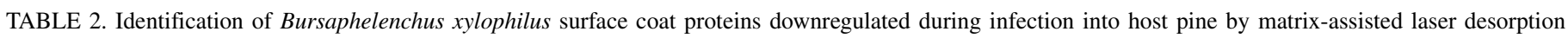
ionization time-of-flight mass spectrometry

\begin{tabular}{|c|c|c|c|c|c|c|c|c|c|}
\hline $\begin{array}{l}\text { Band } \\
\text { no. }\end{array}$ & Cluster ID $^{\mathrm{a}}$ & $\begin{array}{l}\text { Bursaphelenchus } \\
\text { xylophilus protein identity }\end{array}$ & Fold $^{\mathrm{c}}$ & $\begin{array}{l}\text { MW } \\
(\mathrm{kDa})\end{array}$ & $\begin{array}{l}\text { Homologue or similar protein } \\
\text { in other organisms }\end{array}$ & Accession no. ${ }^{\mathrm{d}}$ & $\begin{array}{l}\text { Coverage } \\
(\%)\end{array}$ & $\begin{array}{l}\text { Matched } \\
\text { peptide }\end{array}$ & $\begin{array}{l}\text { MOWSE } \\
\text { score }^{\mathrm{e}}\end{array}$ \\
\hline $7 \mathrm{c} 1$ & $\ldots$ & Unidentified & -11.7 & 13 & $\ldots$ & & $\ldots$ & & $\ldots$ \\
\hline $8 \mathrm{c} 1$ & $\mathrm{n} / \mathrm{a}$ & $\mathrm{n} / \mathrm{a}$ & -3.1 & 12.5 & Unknown (Picea sitchensis) & ABK26292 & 21 & 5 & 69 \\
\hline Dc1 & $\ldots$ & Unidentified & -29.8 & 30 & $\ldots$ & $\ldots$ & $\ldots$ & $\ldots$ & $\ldots$ \\
\hline $\mathrm{Fc} 1$ & $\mathrm{n} / \mathrm{a}$ & $\mathrm{n} / \mathrm{a}$ & -6.2 & 63 & $\begin{array}{l}\text { Hypothetical protein R02D3.3 } \\
\text { (Caenorhabditis elegans) }\end{array}$ & NP_499880 & 12 & 6 & 60 \\
\hline Gc1 & BXC00442_1 & Paramyosin & -3.9 & 78 & Paramyosin (Onchocerca volvulus) & AAA29431 & 14 & 10 & 61 \\
\hline Hc1 & BXC02085_1 & Prohibitin-like molecule & -4.4 & 38.5 & $\begin{array}{l}\text { Prohibitin-like molecule TC-PRO-1 } \\
\text { (Toxocara canis) }\end{array}$ & AAB53231 & 35 & 7 & 86 \\
\hline Kc1 & BXC02983_1 & Enolase 2 & -4.4 & 47.5 & Enolase (C. elegans) & CAH10783 & 30 & 9 & 147 \\
\hline Pc1 & BXC00260_1 & Hypothetical protein & -6.8 & 47.8 & $\begin{array}{l}\text { Hypothetical protein CBG } 21173 \\
\text { (C. briggsae) }\end{array}$ & CAE73664 & 22 & 8 & 88 \\
\hline
\end{tabular}

a Cluster ID given in the B. xylophilus expressed sequence tag database.

b Protein identity by homology with other nematode's entry; $\mathrm{n} / \mathrm{a}=$ not available.

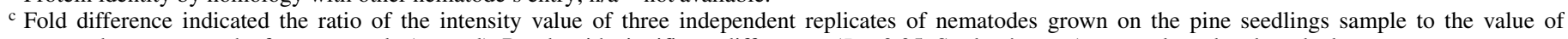
nematodes grown on the fungus sample (control). Bands with significant differences $(P<0.05$; Student's $t$ test) were selected and marked.

${ }^{\mathrm{d}}$ GenBank protein accession number.

e A MOWSE score given by MASCOT search. 
identification, 9 of 29 proteins upregulated (band no. Hp1, Kp1, Lp1, Mp1, Mp3, Mp4, Op1, Sp1, and Vp3) appeared not to be derived from the nematode $B$. xylophilus but, instead, from limited bacterial species (Table 3). The other 20 upregulated proteins were considered to be originated from B. xylophilus; 17 of them (band no. Fp1, Fp2, Fp3, Fp4, Gp1, Hp2, Ip1, Jp1, Mp2, Np1, Np2, Np4, Op2, Qp1, Tp1, Vp2, and Wp1) successfully corresponded to the similar proteins of other organisms by

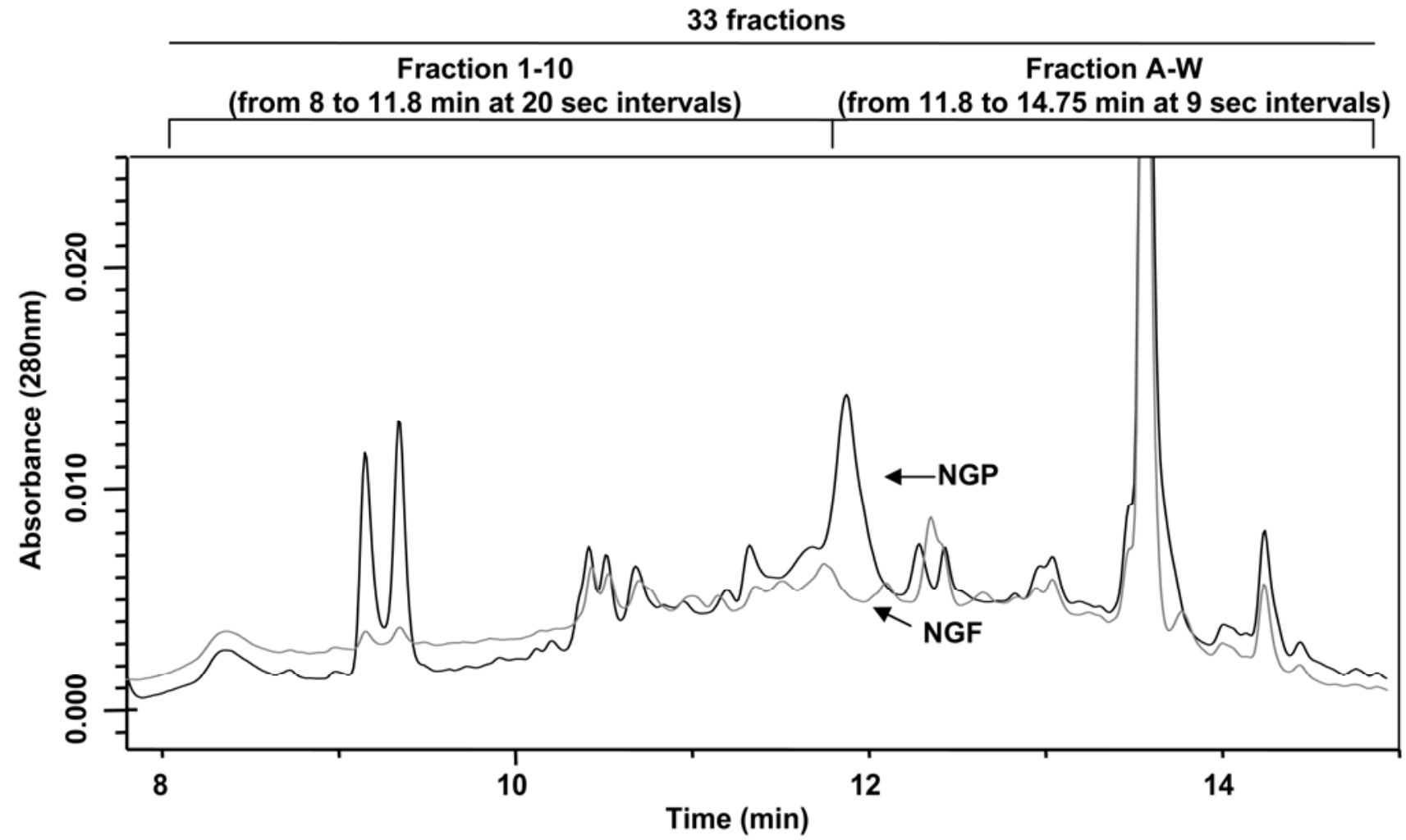

Fig. 2. Reversed-phase high-performance liquid chromatography overlay of chromatograms for Bursaphelenchus xylophilus grown on the fungus Botrytis cinerea (NGF, gray line) and Bursaphelenchus xylophilus grown inside Japanese black pine seedlings (NGP, black line). Thirty-three fractions were split and collected from each separate run to serve sodium dodecyl sulfate polyacrylamide gel electrophoresis.

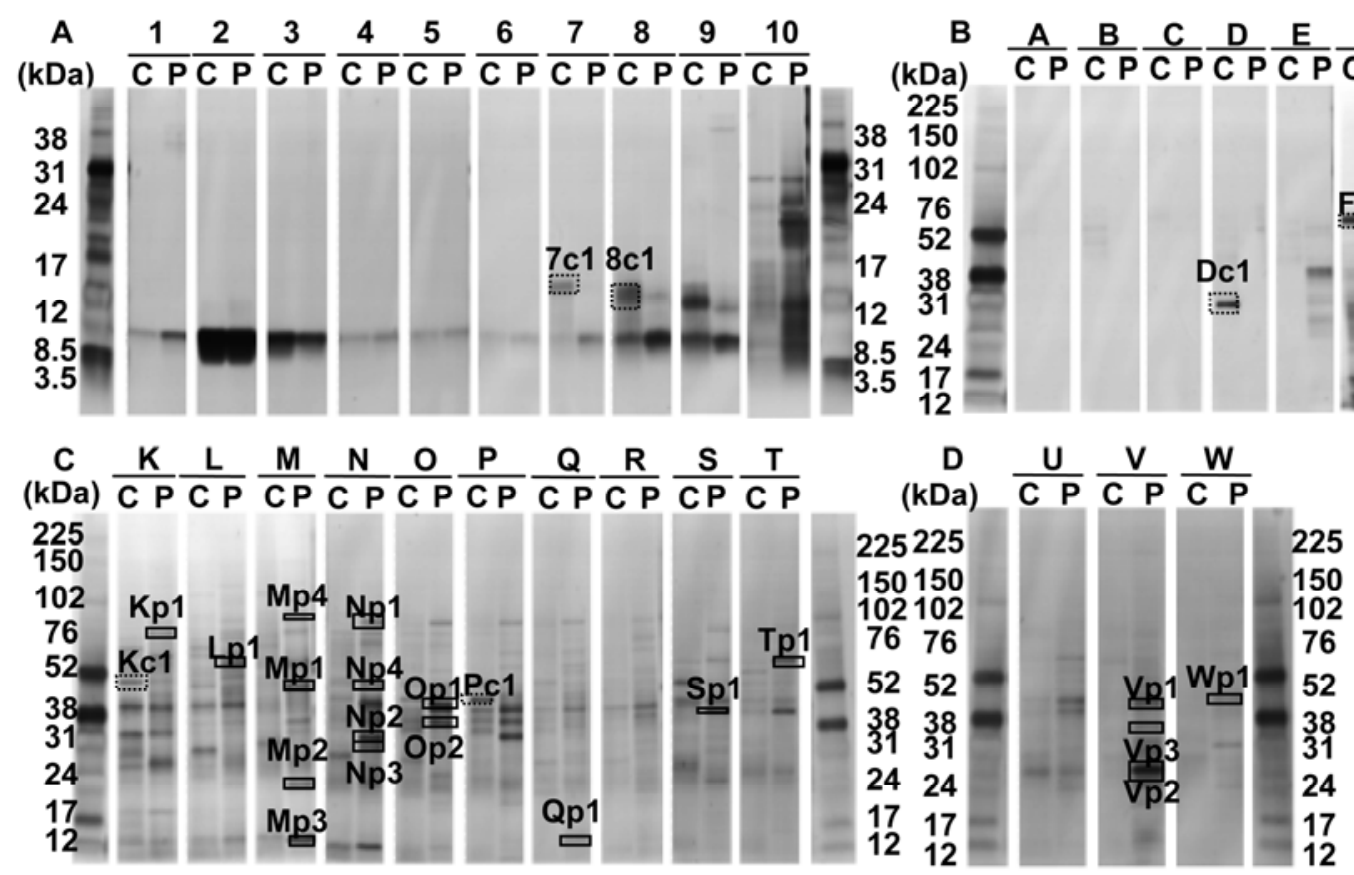

Fig. 3. Comparative analysis with sodium dodecyl sulfate polyacrylamide gel electrophoresis of Bursaphelenchus xylophilus protein-derived reversed-phase highperformance liquid chromatography (RP-HPLC) fractions. Fractions of RP-HPLC: A, 1 to 10; $\mathbf{B}$, A to J; $\mathbf{C}, \mathrm{K}$ to T; and $\mathbf{D}, \mathrm{U}$ to W. Each fraction was run side by side: B. xylophilus grown on the fungus Botrytis cinerea (NGF) on the left, $C$; and B. xylophilus grown inside Japanese black pine (NGP) on the right, $P$. Bands with significant differences $(P<0.05$; student's $t$ test $)$ in band intensity between NGF and NGP were selected and marked. Broken box shows the downregulated proteins in the Japanese black pine seedlings and solid box shows the upregulated proteins in the Japanese black pine seedlings. Numbers described in the upper position of 'C' or 'P' show the fraction number of RP-HPLC. 
MASCOT search (Table 1). In addition, we identified the EST clusters of 12 of 20 upregulated proteins (band no. Fp1, Fp2, Fp3, Hp2, Ip1, Jp1, Np1, Np4, Op2, Qp1, Vp2, and Wp1) by tBLASTn search. However, as for six of eight downregulated proteins (band
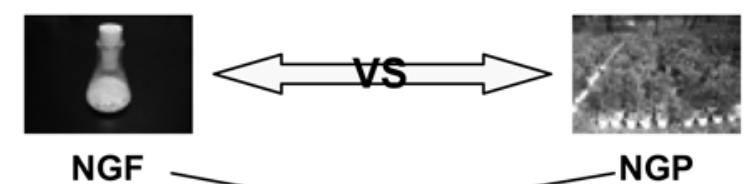

Protein extraction

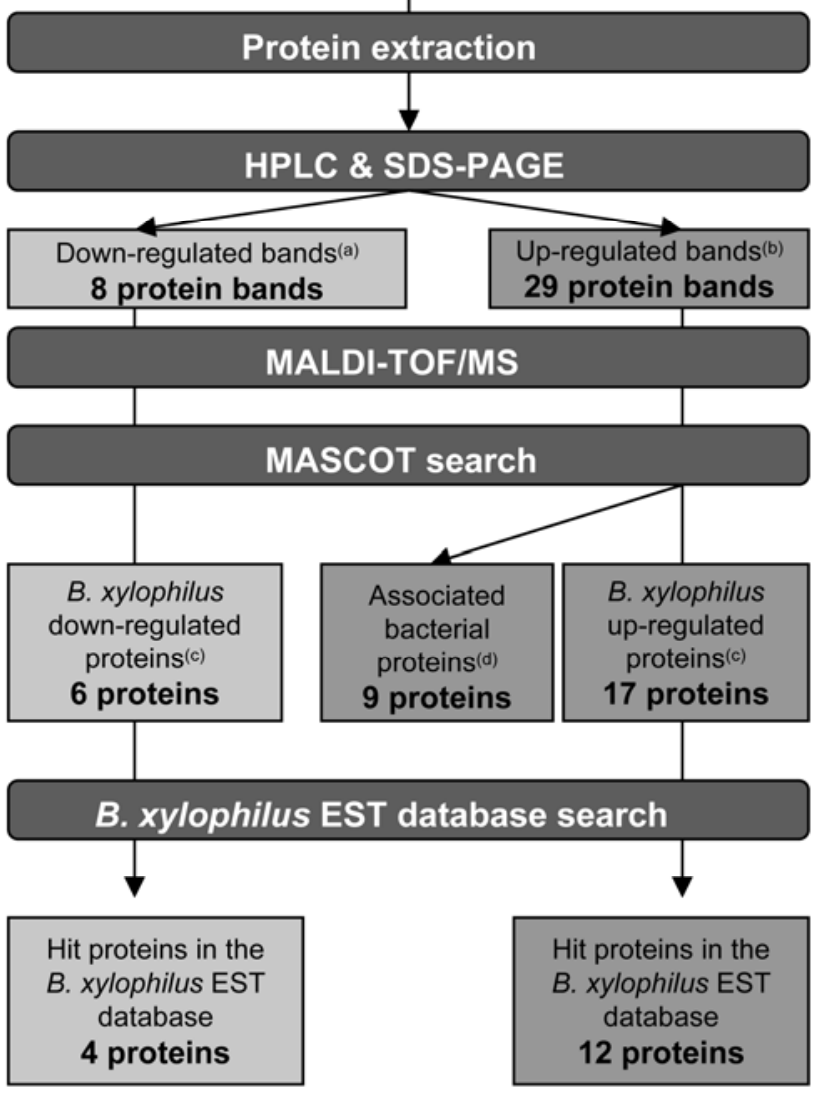

Fig. 4. Overview of the sample process and the results of database searching. (a), Downregulated proteins of the surface coat (SC) proteins of Bursaphelenchus xylophilus grown in the Japanese black pine seedlings (NGP). (b), Upregulated proteins of the SC proteins of B. xylophilus grown in the Japanese black pine seedlings (NGP). (c), Putative B. xylophilus proteins hit to the homologues or similar proteins of other organisms (except for bacteria) by MASCOT search. (d), B. xylophilus-associated bacteria significant hit to the proteins derived from gram-negative bacteria with significant MOWSE scores $(P<0.05)$ by MASCOT search. HPLC $=$ high-performance liquid chromatography; SDS-PAGE = sodium dodecyl sulfate polyacrylamide gel electrophoresis; MALDI-TOF/MS = matrix-assisted laser desorption ionization time of flight mass spectrometry; and EST = expressed sequence tag. no. 8c1, Fc1, Gc1, Kc1, and Pc1), the homologues or similar proteins were successfully detected as being from other organisms by MASCOT search (Table 2). Also, we identified the EST clusters of four of eight downregulated proteins (band no. Gc1, $\mathrm{Hc} 1, \mathrm{Kc} 1$, and Pc1). Five proteins (band no. Hp3, Rp1, Vp1, 7c1, and Dc1) that did not match any data of other organisms by MASCOT search. These five proteins are possibly unique to B. xylophilus.

Upregulated proteins of SC of the PWN. As mentioned above, 20 proteins were upregulated in the SC of PWNs during infection into host pine (Table 1). These include mitochondrial substrate carrier, ADP-ribosylation factor, DnaJ domain protein, GAPDH, gex interacting protein family member, nematode astacin protease family member (NAS), actin, glutathione S-transferase (GST), 14-3-3b protein, MAFBx homolog family member, and seven hypothetical proteins that include two predicted proteins (peptidyl tRNA-hydrase and ATP synthase $\alpha$ subunit). The relative fold-changes were 2.8 to 61.2 .

Downregulated proteins of SC of the PWN. Eight downregulated proteins of PWN were observed during PWN infection into host pine seedlings (Table 2). These include paramyosin, a prohibitin-like molecule, enolase, an unknown protein, and two hypothetical proteins. The significant relative fold-changes were -61.2 to -3.1 .

Proteins of PWN-associated bacteria. In the present study, nine proteins of bacterial origin were found only in the extracted proteins of NGP (Table 3). All of these were successfully identified: glutamine synthetase, heat shock protein 90, ATP synthase subunit $\beta$, nucleoside diphosphate kinase, elongation factor $\mathrm{G}$, elongation factor $\mathrm{Tu}$, and ketol-acid reductoisomerase. These proteins are likely to be surface proteins of bacteria, because the extraction was done with a very mild condition and short period in this study. They all were identified as proteins derived from gram-negative bacteria, and seven of them as proteins of Pseudomonas spp. There are several reports that certain bacteria are associated with the surface of PWN reproducing in pine (40). We also clearly observed (using the SEM) bacteria attached to the surface of PWNs that reproduced only in pine seedlings (Fig. 5).

Immunohistochemical localization by confocal laser-scanning microscope. The immunohistochemical localization of actin, GAPDH, and 14-3-3 protein are shown on the body surface of NGPs (Fig. 6A, B, D, E, G, and H) and NGFs (Fig. 6C, F, and I). In NGPs, all antibodies clearly bound to the body surface of nematodes. In NGFs, however, the antibody bindings were often, but not always, observed onto the body surface of nematodes only for actin and GAPDH (Fig. 6C and F), though the fluorescence signal in NGFs tended to be weaker than in NGPs. In addition, no fluorescence signal was observed for 14-3-3 protein in NGFs (Fig. 6I). There was no significant difference observed in staining pattern among the nematode developmental stages. Negative controls with omission of primary antibody (Fig. 6J) or with preimmune serum (Fig. 6K and L) showed no fluorescence signal.

TABLE 3. Identification of proteins derived from Bursaphelenchus xylophilus-associated bacteria

\begin{tabular}{|c|c|c|c|c|c|c|c|c|}
\hline Band no. & Accession no. ${ }^{\mathrm{a}}$ & Protein identity & Species & $\begin{array}{l}\text { Theoretical } \\
\text { MW (kDa) }\end{array}$ & $\begin{array}{l}\text { Observed } \\
\text { MW (kDa) }\end{array}$ & $\begin{array}{c}\text { Coverage } \\
(\%)\end{array}$ & $\begin{array}{l}\text { Matched } \\
\text { peptides }\end{array}$ & $\begin{array}{c}\text { MOWSE } \\
\text { score }^{b}\end{array}$ \\
\hline Hp1 & BAE44186 & Glutamine synthetase & Pseudomonas taetrolens & 52 & 54 & 22 & 9 & 102 \\
\hline Kp1 & YP_347357 & Heat shock protein 90 & P.fluorescens & 71 & 76 & 10 & 5 & 58 \\
\hline Lp1 & ZP_04616935 & Glutamine synthetase & Yersinia ruckeri & 52 & 52.5 & 18 & 6 & 77 \\
\hline Mp1 & AAY995404 & F0F1 ATP synthase subunit $\beta$ & P.fluorescens & 50 & 49 & 34 & 10 & 126 \\
\hline Mp3 & YP_350333 & Nucleoside diphosphate kinase & P. fluorescens & 15 & 13 & 43 & 4 & 70 \\
\hline Mp4 & YP_001178464 & Elongation factor $\mathrm{G}$ & Enterobacter sp. & 77 & 84 & 17 & 7 & 88 \\
\hline Op1 & YP_350809 & Elongation factor $\mathrm{Tu}$ & P. fluorescens & 44 & 45 & 54 & 16 & 212 \\
\hline Sp1 & YP_351454 & F0F1 ATP synthase subunit $\beta$ & P. fluorescens & 49 & 39.5 & 43 & 12 & 187 \\
\hline Vp3 & YP_262325 & Ketol-acid reductoisomerase & P. fluorescens & 36 & 36 & 20 & 6 & 81 \\
\hline
\end{tabular}

a GenBank protein accession number.

b A MOWSE score given by MASCOT search. 
These results agree with the results of proteome analysis; thus, this immunohistochemical localization authenticated the protein data identified by proteomic approach in this study.

\section{DISCUSSION}

Increase in the total amount of SC proteins during host infection. The amount of total proteins extracted from the PWN body surface greatly differed due to the condition where the PWNs grew (i.e., the total proteins of PWNs reproducing in host pine seedling were much more than those of the PWNs cultured on the fungus) (Fig. 1). Although the proteins extracted in the present study contained a small quantity of those derived from certain PWN-associated bacteria, the results clearly show that a PWN secretes and displays a larger quantity of proteins on its outer surface when it inhabits inside host pine. In fact, the gel band intensities of most SC from PWNs which propagated in pine seedlings (NGPs) were stronger than those of NGFs when the number of PWN individuals that served protein extraction and separation was equally adjusted (data not shown). Furthermore, no upregulated proteins identified here had any plant matches as their top hit in MASCOT search. These results indicate that the cause of the increase in total amount of proteins extracted was solely the increase of the amount of PWN SC proteins. This finding supports the idea that the SC of PWNs should be related to the infection process of PWD as the host-parasite interface. In addition, when the ultrastructure of the outer surface of the PWN body was observed by SEM, no clear difference was found between NGFs and NGPs (Fig. 5). Thus, the surface change in the PWN which occurs in entering into the host pine seems to be at the molecular level, not accompanied by any changes in the surface structure.

Overview of SC protein identification results. In the present study, $\approx 100$ proteins were successfully separated by a special twodimensional protein separation system that combines RP-HPLC with SDS-PAGE. As a result, 17 upregulated and 6 downregulated proteins derived from PWN infesting pine seedlings were successfully annotated with protein functions. In addition, nine proteins derived from PWN-associated bacteria were identified. We classified some of the identified proteins into four categories based on their biological properties which may be concerned with PWD—regulation of ROS production, ROS scavenger, protein with an unexpected role, and proteins of PWN-associated bacteria-and advance the following discussions according to these classifications.

Regulation of ROS production. The 14-3-3 proteins have been well known as kinase and phosphatase. However, there are also several reports that 14-3-3 proteins are involved in ROS regulation and apoptosis in recent years $(9,19,38)$. The gene coding 14-3-3 protein has been identified in several unicellular and multicellular parasites. Interestingly, some reports suggested a shift in 14-3-3 gene expression during transition to the successive parasitic stages in some parasites (33). However, little is known about associated functionality or production patterns of the corresponding proteins in these parasites. Regarding plantparasitic nematodes, it was reported that Meloidogyne incognita secreted 14-3-3b protein from the stylet of infective juveniles $(2,15)$. However, in plants, a 14-3-3 protein regulates the plasma membrane $\mathrm{H}^{+}$-ATPase in response to pathogen attack, and also regulates the production of ROS in plant-pathogen interactions $(9,14)$.

ROS scavenger. Two SC proteins were identified that could potentially act as ROS scavengers, including GST and GAPDH. GST is a kind of antioxidant enzyme, and it is known that various parasites overexpress GST during the infection process $(5,22)$. Furthermore, GST was reported to localize on the surface of various parasites, and the localization might allow the parasites to protect themselves from oxidative damage on the cuticular membranes (30). GAPDH is generally regarded as a housekeeping glycolytic enzyme. However, many reports suggest that GAPDH is a multifunctional protein that is involved in various subcellular processes in animals $(34,35)$. One of these functions is cellular redox regulation. Baek et al. (1) suggested that GAPDH had a direct ROS scavenging activity. Thus, there is a fair possibility that GAPDH localized on the surface of PWNs acts as a ROS scavenger, although further study will be needed to confirm the precise function of this molecule.

Protein with an unexplained role. Another interesting PWN SC protein with unexplained roles is the NAS protein. NAS proteins are metalloproteases present in various organisms which serve multiple physiological functions $(7,26)$. However, information about the detailed functions of NAS proteins is very limited. In the animal parasites, several reports indicated that the metalloprotease secreted from parasites was a key molecule in its infection process $(4,23,37)$. Williamson et al. (37) reported that the dog hookworm Ancylostoma caninum secretes an astacin-like metalloprotease in the infective stage during host migration, and suggested that this metalloprotease localized on the outer surface of hook worm was critical for its invasion process.

Proteins of PWN-associated bacteria. In upregulated proteins, nine proteins were not identified as PWN proteins because they best matched bacterial proteins by MASCOT search and because no significant tBLASTn similarity was detected to any available amino acid sequences of $B$. xylophilus using ForestGEN server. Also, the deduced origins of the bacterial proteins indicated by the results of MASCOT search were very limited, mostly Pseudomonas spp. It has been reported that PWNs are accompanied by some bacteria on their outer surface during reproduction in the pine tree, of which major bacterial species are Pseudomonas $(12,40)$. Also, in the present study, the PWN might be accompanied by some bacteria on its outer surface when it was propagated in the pine seedlings and, therefore, the protein derived from the PWN-associated bacteria might be extracted and identified, although the PWN mass collected from the seedlings was vigorously washed many times before extraction of SC protein. In fact, some bacteria attached to the PWN outer surface were observed by SEM (Fig. 5). Zhao et al. (40) and Han et al. (12) suggested that the complex infection of PWNs and their

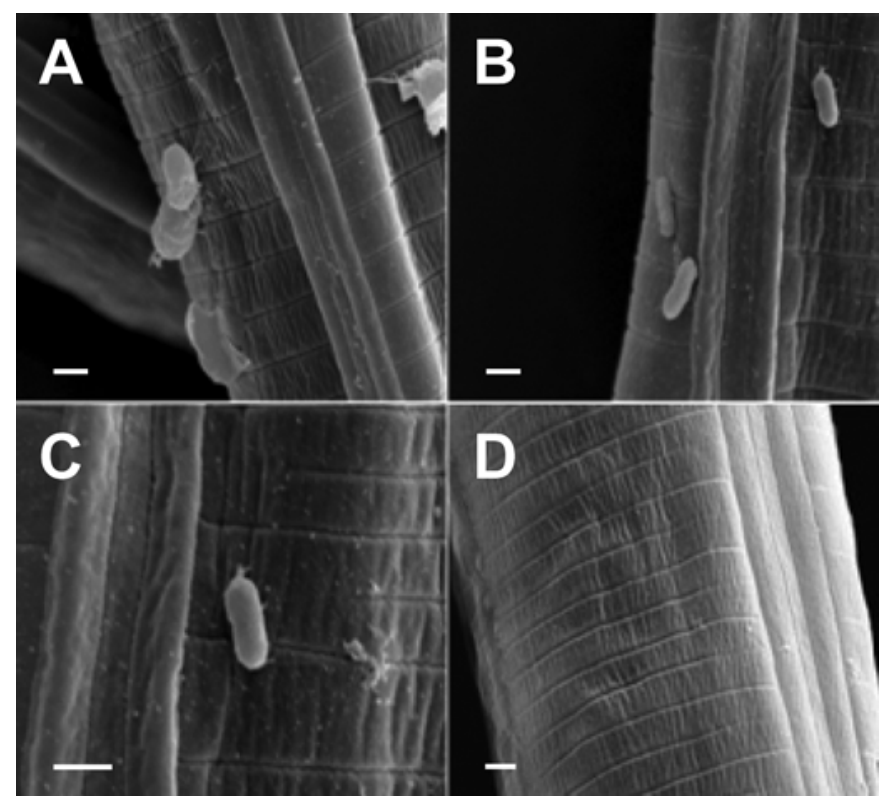

Fig. 5. Scanning electron microscope images of $\mathbf{A}, \mathbf{B}$, and $\mathbf{C}$, ultrastructure of the body surface of the pine wood nematode (PWN) grown in the pine seedlings and its associated bacteria attaching to the surface of the PWN body; D, ultrastructure of the body surface of PWN grown on the fungus. Scale bars: $1.0 \mu \mathrm{m}$. 


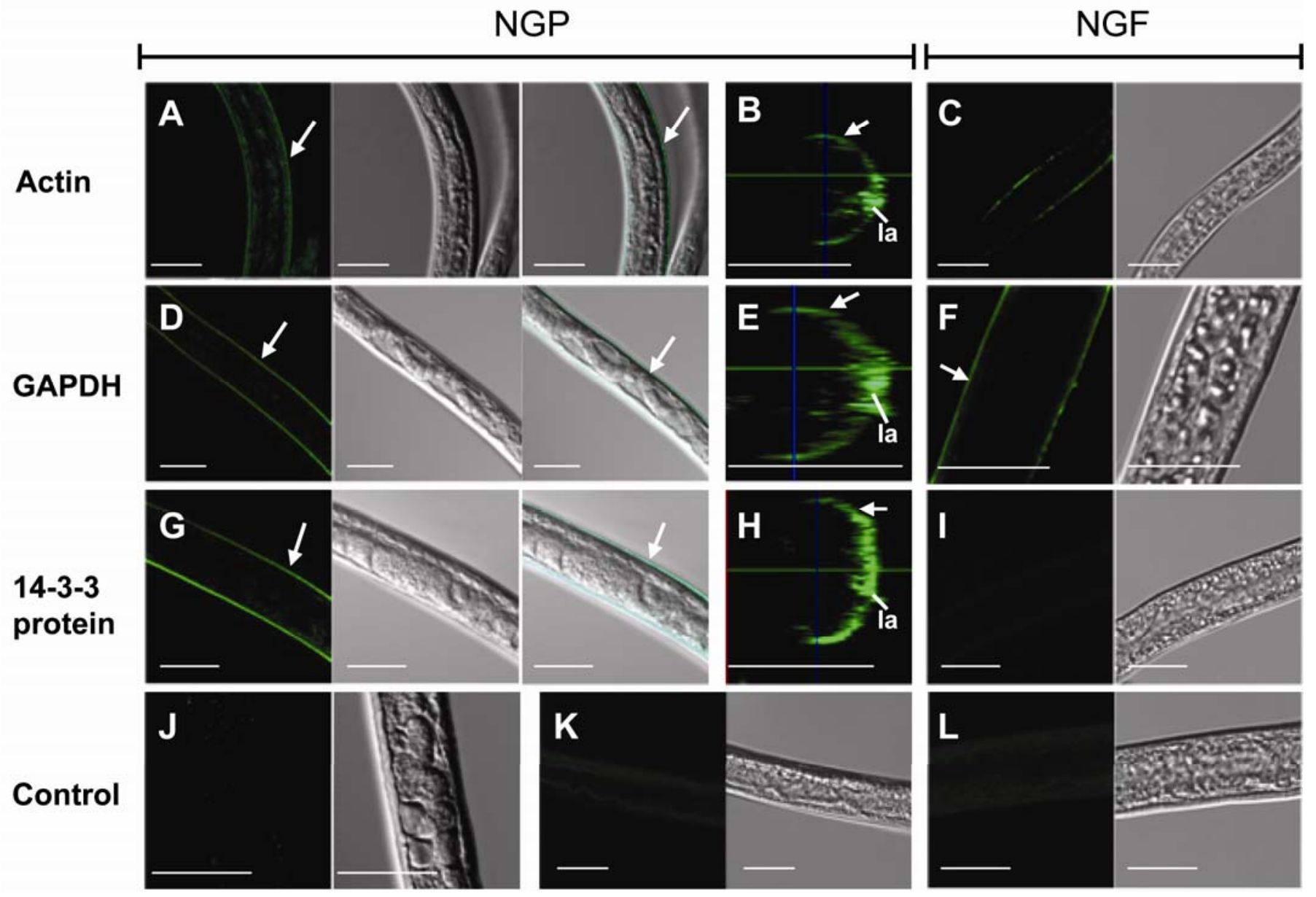

Fig. 6. Immunohistochemical localization of actin, glyceraldehydes-3-phosphate dehydrogenase (GAPDH) and 14-3-3 protein on the body surface of $\mathbf{C}$, F, and I, Bursaphelenchus xylophilus grown on the fungus and $\mathbf{A}, \mathbf{B}, \mathbf{D}, \mathbf{E}, \mathbf{G}$, and $\mathbf{H}$, in the pine seedlings. The B. xylophilus were stained with primary antibody against actin, GAPDH, or 14-3-3 protein followed by fluorescein-isothiocyanate-conjugated secondary antibody. Negative control experiments were also carried out by incubating the B. xylophilus without primary antibody $(\mathbf{J})$ or with rabbit preimmune serum $(\mathbf{K}$ and $\mathbf{L})$. Fluorescence microscopy images with black background (the far left image in $\mathbf{A}, \mathbf{C}, \mathbf{D}, \mathbf{F}, \mathbf{G}, \mathbf{I}, \mathbf{J}, \mathbf{K}$, and $\mathbf{L}$ ); normal light microscope images (the middle image in $\mathbf{A}, \mathbf{D}$, and $\mathbf{G}$ and the right image in $\mathbf{C}, \mathbf{F}, \mathbf{I}$, $\mathbf{J}, \mathbf{K}$, and $\mathbf{L}$ ); fluorescence microscopy images with white background (the far right image in $\mathbf{A}, \mathbf{D}, \mathbf{G})$. Transverse image of B. xylophilus treated with anti-actin antibody $(\mathbf{B})$; anti-GAPDH antibody $(\mathbf{E})$; anti-14-3-3 protein antibody $(\mathbf{H})$. Contrast of images $\mathbf{C}$ and $\mathbf{F}$ were increased to make it easier to understand, while the contrast of all the other images was same. White arrows show each protein clearly localized on the surface of B. xylophilus. Scale bars: $20 \mu \mathrm{m} ; 1 \mathrm{a}=1$ ateral field.

accompanying bacteria is responsible. However, it should not be denied at present that these proteins, which have bacterial proteins as their best matches, may possibly be derived from the PWN itself, because the PWN acquired several genes by horizontal gene transfer from bacteria. More studies are needed to determine the exact origins of these proteins.

Possible roles of the PWN SC proteins in the development of PWD. In PWD, in the pine trees infected by PWNs, the excess ROS production and the leakage of electrolytes have been observed at the initial stage of infection (21). Some researchers hypothesized that these abnormal regulations of ROS productions may finally lead the pine trees to death $(13,39)$. That is, it can be speculated that the regulation and evasion of ROS might be the key point in the disease. In the present study, we revealed that the 14-3-3 protein known as a potential regulator of ROS was localized on the surface of PWNs during host pine infection. Furthermore, two potential ROS scavengers, including GST and GAPDH, were identified. These proteins may not be only an antioxidative barrier but also a fundamental factor to establish and let persist the host-parasite relationship. The results of this study suggest that the PWN has a kind of mechanism to evade or modulate the host defense responses, and one of the main sites that serve such an important function can be the SC of PWNs, which stands at the interface with the plant immune response. Further analysis of these interesting proteins will be required to verify our hypotheses.
Conclusions. Here, we provide the first report that focused on the SC proteins and thoroughly identified those of plant-parasitic nematodes by proteome analysis. In the present study, we may say that most of the major SC proteins of the PWN were successfully identified using PMF by improving the protein separation system and collecting a large quantity of samples, although it could enable us to perform large-scale analysis and identify the protein with more accuracy by using tandem MS. In fact, many of the PWN SC proteins identified here corresponded to the surface proteins of the animal parasite Schistosoma bovis (29). The PWN SC proteins identified contained a potential regulator of ROS production, and ROS scavengers. These functions of PWN SC proteins seem to be essential for establishing their infection and causing sudden death of host pine trees by the PWD. Further studies are needed to determine whether these SC proteins of the PWN are really involved in pathogenesis. This work will contribute to revealing the biological roles of the SC proteins of plant-parasitic nematodes and solving puzzles behind the PWD mechanisms.

\section{ACKNOWLEDGMENTS}

This study was supported by a grant-in-aid for JSPS Fellows and partly supported by a grant-in-aid for Young Scientists (B) (no. 21780147). We thank J. Miwa and K. Hasegawa, Chubu University, Japan, for their technical support; J. R. Sutherland, Canada, for linguistic correction; and 
K. Ichimura and K. Tsugawa, Kyoto University, Japan, for their helpful support concerning the sample collection.

\section{LITERATURE CITED}

1. Baek, D., Jin, Y., Jeong, J. C., Lee, H. J., Moon, H., Lee, J., Shin, D., Kang, C. H., Kim, D. H., Nam, J., Lee, S. Y., and Yun, D.J. 2008. Suppression of reactive oxygen species by glyceraldehyde-3-phosphate dehydrogenase. Phytochemistry 69:333-338.

2. Bellafiore, S., Shen, Z., Rosso, M. N., Abad, P., Shih, P., and Briggs, S. P. 2008. Direct identification of the Meloidogyne incognita secretome reveals proteins with host cell reprogramming potential. PLoS Pathog. 4:e1000192. doi:10.1371/journal.ppat.1000192.

3. Bird, A. F. 2004. Surface adhesion to nematodes and its consequences. Pages 295-329 in: Nematology: Advances and Perspectives. Volume 1: Nematode Morphology, Physiology and Ecology. Z. X. Chen, S. Y. Chen, and D. W. Dickson, eds. CAB International, Wallingford, UK.

4. Borchert, N., Becker-Pauly, C., Wagner, A., Fischer, P., Stöcker, W., and Brattig, N. W. 2007. Identification and characterization of onchoastacin, an astacin-like metalloproteinase from the filaria Onchocerca volvulus. Microbiol. Infect. 9:498-506.

5. Brophy, P. M., Ben-Smith, A., Brown, A., Behnke, J. M., and Pritchard, D. I. 1995. Differential expression of glutathione S-transferase (GST) by adult Heligmosomoides polygyrus during primary infection in fast and slow responding hosts. Int. J. Parasitol. 25:641-645.

6. Christie, J. R., and Perry, V. G. 1951. Removing nematodes from soil. Proc. Helminthol. Soc. Wash. 18:106-108.

7. Culley, F. J., Brown, A., Conroy, D. M., Sabroe, I., Pritchard, D. I., and Williams, T. J. 2000. Eotaxin is specifically cleaved by hookworm metalloproteases preventing its action in vitro and in vivo. J. Immunol. 165:6447-6453.

8. Curtis, R. H. C. 2007. Plant parasitic nematode proteins and the hostparasite interaction. Brief. Funct. Genomics Proteomics 6:50-58.

9. Elmayan, T., Fromentin, J., Riondet, C., Alcaraz, G., Blein, J. P., and Simon-Plas, F. 2007. Regulation of reactive oxygen species production by a 14-3-3 protein in elicited tobacco cells. Plant Cell Environ. 30:722-732.

10. Gravato-Nobre, M. J., and Evans, K. 1998. Plant and nematode surfaces: their structure and importance in host-parasite interactions. Nematologica 44:103-124.

11. Gravato-Nobre, M. J., McClure, M. A., Dolan, L., Calder, G., Davies, K. G., Mulligan, B., Evans, K., and Von Mende, N. 1999. Meloidogyne incognita surface antigen epitopes in infected Arabidopsis roots. J. Nematol. 31:212-223.

12. Han, Z. M., Hong, Y. D., and Zhao, B. G. 2003. A study on pathogenicity of bacteria carried by pine wood nematodes. J. Phytopathol. 151:683-689.

13. Iwahori, H., and Futai, K. 1993. Lipid peroxidation and ion exudation of pine callus tissues inoculated with pinewood nematodes. Jpn. J. Nematol. 23:79-89.

14. Jahn, A. T., Fuglsang, A., Olsson, I. M., Bruntrup, D. B., Collinge, D., Volkmann, M., Sommarin, M., Palmgren, M. G., and Larsson, C. 1997. The 14-3-3 protein interacts directly with the C-terminal region of the plant plasma membrane $\mathrm{H}^{+}$-ATPase. Plant Cell 9:1805-1914.

15. Jaubert, S., Laffaire, J. B., Ledger, T. N., Escoubas, P., Amri, E. Z., Abad, P., and Rosso, M. N. 2004. Comparative analysis of two 14-3-3 homologues and their expression pattern in the root-knot nematode Meloidogyne incognita. Int. J. Parasitol. 34:873-880.

16. Kikuchi, T., Aikawa, T., Kosaka, H., Pritchard, L., Ogura, N., and Jones, J. T. 2007. Expressed sequence tag (EST) analysis of the pine wood nematode Bursaphelenchus xylophilus and B. mucronatus. Mol. Biochem. Parasitol. 155:9-17.

17. Kikuchi, T., Jones, J. T., Aikawa, T., Kosaka, H., and Ogura, N. 2004. A family of glycosyl hydrolase family 45 cellulases from the pine wood nematode Bursaphelenchus xylophilus. FEBS Lett. 572:201-205.

18. Kikuchi, T., Shibuya, H., Aikawa, T., and Jones, J. T. 2006. Cloning and Characterization of pectate lyases secreted by the pine wood nematode Bursaphelenchus xylophilus. Mol. Plant-Microbe Interact. 19:280-287.

19. Kim, J. S., Diebold, B., A. Babior, B. M., Knaus, U. G., and Bokock, G. M. 2007. Regulation of Nox 1 activity via protein kinase A-mediated phosphorylation of NoxA1 and 14-3-3 binding. J. Biol. Chem. 282:34787-34800.

20. Kiyohara, T., and Tokushige, Y. 1971. Inoculation experiments of a nematode, Bursaphelenchus sp., onto pine trees. J. Jpn. For. Soc. 53:210218.

21. Komatsu, M., and Suzuki, K. 2006. Electrolyte leakage from necrotizing tissues of Japanese black pine (Pinus thunbergii) inoculated with pine wood nematodes. Tree For. Health 10:25-35.

22. LoVerde, P. T. 1998. Do antioxidants play a role in schistosome hostparasite interaction? Parasitol. Today 14:285-289.

23. Lun, H. M., Mak, C. H., and Ko, R. C. 2003. Characterization and cloning of metallo-proteinase in the excretory/secretory products of the infectivestage larva of Trichinella spiralis. Parasitol. Res. 90:27-37.

24. Maizels, R. M., Robertson, B. D., Blaxter, M. L., and Selkirk, M. E. 1991. Page 14 in: Parasite Antigens, Parasite Genes. A Laboratory Manual for Molecular Parasitology. Cambridge University Press, Cambridge.

25. Mezhoud, K., Praseuth, D., Puiseux-Dao, S., François, J. C., Bernard, C., and Edery, M. 2008. Global quantitative analysis of protein expression and phosphorylation status in the liver of the medaka fish (Oryzias latipes) exposed to microcystin-LR I. Balneation study. Aquat. Toxicol. 86:166-175.

26. Möhrlen, F., Hutter, H., and Zwilling, R. 2003. The astacin protein family in Caenorhabditis elegans. Eur. J. Biochem. 270:4909-4920.

27. Mota, M., Braasch, H., Bravo, M. A., Penas, A. C., Burgermeister, W., Metge, K., and Sousa, E. 1999. First report of Bursaphelenchus xylophilus in Portugal and in Europe. Nematology 1:727-734.

28. Odani, K., Sasaki, S., Nishiyama, Y., and Yamamoto, N. 1985. Early symptom development of the pine wilt disease by hydrolytic enzymes produced by the pine wood nematodes-cellulase as a possible candidate of the pathogen. J. Jpn. For. Soc. 67:366-372.

29. Pérez-Sánchez, R., Valero, M. L., Ramajo-Hernández, A., Siles-Lucas, M., Ramajo-Martín, V., and Oleaga, A. 2008. A proteomic approach to the identification of tegumental proteins of male and female Schistosoma bovis worms. Mol. Biochem. Parasitol. 161:112-123.

30. Selkirk, M. K., Smith, V. P., Thomas, G. R., and Gounaris, K. 1998. Resistance of filarial nematode parasites to oxidative stress. Int. J. Parasitol. 28:1315-1332.

31. Sharon, E., Spiegel, Y., Salomon, R., and Curtis, R. H. C. 2002. Characterization of Meloidogyne javanica surface coat with antibodies and their effect on nematode behaviour. Parasitology 125:177-185.

32. Shinya, R., Takeuchi, Y., Miura, N., Kuroda, K., Ueda, M., and Futai, K. 2009. Surface coat proteins of the pine wood nematode, Bursaphelenchus xylophilus: profiles of stage- and isolate-specific characters. Nematology 11:429-538.

33. Siles-Lucas, M. M., and Gottstein, B. 2003. The 14-3-3 protein: A key molecule in parasites as in other organisms. Trends Parasitol. 19:575-581.

34. Sirover, M. A. 1999. New insights into an old protein: The functional diversity of mammalian glyceraldehyde-3-phosphate dehydrogenase. Biochim. Biophys. Acta 1432:159-184.

35. Sirover, M. A. 2005. New nuclear functions of the glycolytic protein, glyceraldehyde-3-phosphate dehydrogenase, in mammalian cells. J. Cell Biochem. 95:45-52.

36. Spiegel, Y., and McClure, M. A. 1995. The surface coat of plant-parasitic nematodes: Chemical composition, origin, and biological role- - a review. J. Nematol. 27:127-134.

37. Williamson, A. L., Lustigman, S., Oksov, Y., Deumic, V., Plieskatt, J., Mendez, S., Zhan, B., Bottazzi, M. E., Hotez, P. J., and Loukas, A. 2006. Ancylostoma caninum MTP-1, an astacin-like metalloprotease secreted by infective hookworm larvae, is involved in tissue migration. Infect. Immun. 74:961-967.

38. Xing, H., Zhang, S., Weinheimer, C., Kovacs, A., and Muslin A. J. 2000. 14-3-3 proteins block apoptosis and differentially regulate MAPK cascades. EMBO J. 19:349-358.

39. Yamada, T. 2008. Biochemical responses in pine trees affected by pine wilt disease. Pages 223-234 in: Pine Wilt Disease. B. G. Zhao, K. Futai, J. R. Sutherland, and Y. Takeuchi, eds. Springer, Tokyo.

40. Zhao, B., Wang, H., Han, S. F., and Han, Z. M. 2003. Distribution and pathogenicity of bacteria species carried by Bursaphelenchus xylophilus in China. Nematology 5:899-906. 